\title{
P. Chambat, P. H. Neyret, M. Bonnin, D. Dejour, J. C. Panisset, R. Badet, E. Servien, B. Sonnery-Cottet, J. Barth, N. Bonin: 13èmes Journées lyonnaises de chirurgie du genou Le genou et le sport $\mathrm{Du}$ ligament à la prothèse
}

Sauramps Médical 2008, 278 pages, 40.00 $€$, ISBN: 978-2-84023-590-3

\section{Pierre Kehr}

Received: 15 August 2009 / Accepted: 18 August 2009 / Published online: 2 September 2009

(C) Springer-Verlag 2009

Quels sont les effets de la chirurgie du genou sur la pratique du sport? C'est le but de ce livre, qu'il s'agisse de lésions ligamentaires, osseuses ou cartilagineuses; traumatiques, dysplasiques ou dégénératives.

Le livre est divisé en trois partie s: la rotule, le LCA et l'arthrose. Il est néanmoins à remarquer qu'une grande part du livre est consacré à la reconstruction du LCA. D'autres chapitres évaluent les résultats fonctionnels y compris sportifs.
Au total un point détape intéressant pour savoir ce qui se fait à l'heure actuelle et avec quels résultats.

No funds were received in support of this study.

Alain Graftiaux Strasbourg

P. Kehr $(\square)$

Strasbourg, France

e-mail: kehrpier@aol.com 